\title{
Elective affinities
}

\author{
A feasibility study for the systematic generation of affinity reagents to human proteins \\ provides an opportunity to test the merits of recombinant affinity reagents.
}

Antibodies are perhaps the most ubiquitous reagents in biological research. Yet their production relies on a natural immune reaction, which limits feasible targets, is costly, time-consuming and unpredictable. One would expect molecular biology to deliver a better solution. And indeed, from antibody fragments to non-immunoglobulin scaffolds, a variety of alternative, recombinant affinity reagents have been developed, which are generated by in vitro selection from molecular libraries instead of immunization (see, for example, Research Highlight, p. 861).

Although attractive for therapeutic applications, for which in vitro manufacturing is an unconditional advantage, recombinant reagents have not been broadly embraced as tools in biological research. They are not widely available and there is little evidence that, for research purposes, they are really on par with antibodies-despite theoretical advantages such as better control of the epitope, nonreliance on target immunogenicity and propensity to recognize conformational epitopes.

An ongoing study described on page 854 offers an interesting opportunity to put recombinant affinity reagents to the test. The primary aim of this pilot project is to evaluate the feasibility of systematically making quality affinity reagents to human proteins, in the context of large-scale proteomic investigations. But because of its setup, this pilot project can also begin to address the important question of how different recombinant affinity reagents compare to each other and to conventional antibodies in various applications.

The target set of antigens - a group of $\mathrm{SH} 2$-containing proteins - is relatively small and, being composed of closely related proteins from a single family, limits the scope of the project. Nevertheless, this pilot project benefits from the participation of experts in the affinity reagent field, and thanks to a collaboration with the Structural Genomic Consortium, all antigens are being provided in two forms, each particularly suited to raise different types of affinity reagents: properly folded protein domains and partially denatured peptides.

Even with the unique opportunity offered by the availability of both kinds of protein targets and by the participation of groups specialized in different affinity reagents, comparing affinity reagents as part of this project will not be trivial — in particular because state-of-theart production procedures for the various reagents are not on par. Specifically, increasing the throughput of in vitro selection has proven complicated, and the current production capacity for recombinant reagents does not rival what can be obtained for conventional antibodies in highly automated pipelines. For example, the Human Protein Atlas in Sweden has released in August the latest version of its collection of polyclonal antibodies, totaling 6,120 antibodies. They have also developed a web portal to share antibody validation data (see Research Highlight, p. 860). Meanwhile, Monash University in Australia has announced the opening of a high-tech monoclonal antibody production facility.

As part of the pilot project, there is little doubt that these established production pipelines will rapidly deliver antibodies. But it will be a taller order for producers of recombinant affinity reagents. Given the absence of specific funding for this grass-roots project, recombinantreagent producers are on the back foot. One of the challenges for the coordinators will be to give every participant group a fair chance to contribute a meaningful number of reagents without delaying the project unduly.

Similarly, the existing well-controlled validation pipelines for affinity reagents are geared toward conventional antibodies. If this pilot project is to also compare the performance of different affinity reagents, the evaluation will need to include assays in which recombinant reagents have a theoretical advantage, such as applications that require recognition of conformational epitopes. That, in turn, will require the participation of end-users. It is therefore critical that the community of biologists interested in SH2-containing proteins responds to the call for participation and actively engages in evaluating the variety of reagents that the pilot project will produce.

For these volunteer end-users, there could be a larger, long-term benefit. If it turns out that some recombinant reagents have an edge for some specific applications, this demonstration could go a long way toward focusing additional efforts to overcome their limitations and make them broadly available as research reagents. In particular, developments would be needed to increase production capacity and to generate appropriate starting antigens. Some issues related to intellectual property rights may also need addressing — probably via agreements that distinguish clinical and research applications.

There is little incentive at the moment to invest in solving these problems, but an experimental demonstration could change that. With the proper momentum, the problems can probably be addressed. The open, grassroots pilot project has the potential to generate that momentum. We wish its participants good luck. 